\title{
Impact of preoperative hand grip strength on morbidity following gastric cancer surgery
}

\author{
Tsutomu Sato $^{1,2}$ - Toru Aoyama ${ }^{1,2} \cdot$ Tsutomu Hayashi $^{2} \cdot$ Kenki Segami $^{1,2}$ • \\ Taiichi Kawabe $^{1,2} \cdot$ Hirohito Fujikawa ${ }^{1,2}$ - Takanobu Yamada ${ }^{2}$. Naoto Yamamoto ${ }^{2}$. \\ Takashi Oshima $^{2} \cdot$ Yasushi Rino $^{2} \cdot$ Munetaka Masuda $^{2} \cdot$ Takashi Ogata $^{1} \cdot$ \\ Haruhiko $\mathrm{Cho}^{1} \cdot$ Takaki Yoshikawa ${ }^{1,2}$
}

Received: 10 June 2015/ Accepted: 28 September 2015/Published online: 14 October 2015

(c) The International Gastric Cancer Association and The Japanese Gastric Cancer Association 2015

\begin{abstract}
Background Sarcopenia is a decrease in both muscle mass and strength. It remains unclear whether sarcopenia is associated with morbidity after gastric cancer surgery. This study evaluated the impact of sarcopenia on the morbidity of gastric cancer surgery.

Methods A total of 293 gastric cancer patients who underwent curative surgery between May 2011 and June 2013 were retrospectively examined. Patients with performance status 3 or 4 were excluded. Preoperative lean body mass (LBM) was evaluated by bioelectrical impedance analysis and expressed as LBM index. Preoperative muscle function was measured by hand grip strength (HGS). The cutoff values were the gender-specific lowest $20 \%$. Grade 2 or higher morbidities, as retrospectively evaluated by the Clavien-Dindo classification, were obtained from the patient record. The risk factors for morbidity were examined by univariate and multivariate analyses.

Results Morbidity was observed in 39 patients (13.3\%), including 7 with pancreatic leakage, 12 with anastomotic leakage, and 4 with intraabdominal abscesses, but no mortality was observed. The univariate analysis showed that male gender, total gastrectomy, splenectomy, and a low HGS were significant risk factors for morbidity. A low
\end{abstract}

T. Sato and T. Aoyama contributed equally to this manuscript.

Takaki Yoshikawa

yoshikawat@kcch.jp

1 Department of Gastrointestinal Surgery, Kanagawa Cancer Center, 2-3-2 Nakao, Asahi-ku, Yokohama-Shi, Kanagawa-ken 241-8515, Japan

2 Department of Surgery, Yokohama City University, 3-9 Fukuura, Kanazawa-ku, Yokohama-Shi, Kanagawa-ken 236-0004, Japan
LBM was not a significant risk factor. A low HGS, male gender, and total gastrectomy remained significant in the multivariate analysis.

Conclusions A low hand grip strength was a significant risk factor for morbidity after gastric cancer surgery. The importance of the hand grip strength as a risk factor should be examined in future prospective studies.

Keywords Gastric cancer - Sarcopenia - Hand grip strength $\cdot$ Clavien-Dindo classification $\cdot$ Morbidity (3-5)

\section{Introduction}

Surgery is the mainstay of treatment for gastric cancer, and complete tumor removal is essential to cure gastric cancer. D2 gastrectomy is now accepted as the standard surgery not only in Japan but also in Europe and the United States (USA) [1, 2]. D2 surgery is a safe procedure in Japan; however, it sometimes leads to lethal complications. Several factors, such as older age, the American Society of Anesthesiologists (ASA) score, obesity (body mass index $\geq 25$ ), higher visceral fat area, and multiple comorbidities have previously been identified as risk factors for gastric cancer surgery [3-6].

Sarcopenia may be another important candidate risk factor. Sarcopenia is a syndrome characterized by a progressive and generalized decrease in the amount and strength of skeletal muscle. Sarcopenia is an age-related change, but it is also observed in young adults under certain conditions. Sarcopenia has been shown to be associated with a poor outcome, increased hospital stay, and increased treatment toxicity in several malignancies [7-10]. More recently, sarcopenia, defined by reduced muscle mass, was reported to be a significant risk factor for postoperative complications 
following colorectal cancer surgery and metastatic liver resection $[11,12]$. However, muscle mass does not correlate linearly with muscle strength. Therefore, the extent of sarcopenia and its clinical value should be evaluated using both muscle mass and muscle strength. In the present study, we evaluated bioelectrical impedance analysis (BIA) as a measure of muscle mass and hand grip strength (HGS) as a measure of muscle strength. Although BIA is not a direct measurement of muscle mass different from image analysis, BIA is safe and convenient. Moreover, it remains unclear whether sarcopenia is also associated with the morbidity of gastric cancer surgery.

We herein investigated the impact of sarcopenia, as characterized by muscle mass or strength, on the morbidity of gastric cancer surgery.

\section{Methods}

\section{Selection of patients}

The study subjects were selected from the medical records of 315 consecutive patients who underwent gastrectomy for gastric adenocarcinoma at Kanagawa Cancer Center from May 2011 to June 2013, based on whether they met the following criteria: (1) pathologically confirmed adenocarcinoma of the stomach, (2) had undergone D2 or D1+ gastrectomy for gastric cancer as a primary treatment, (3) R0 or R1 resection was achieved, (4) Eastern Cooperative Oncology Group (ECOG) performance status was 0-2 before surgery, (5) no multiple primary cancer required simultaneous other visceral resection, and (6) bioelectrical impedance analysis (BIA) and hand grip strength (HGS) were measured before surgery. The present study excluded 2 patients who underwent R2 resection, 5 patients with double cancer, and 15 patients for whom there were no data on skeletal muscle mass or strength. Finally, 293 patients were enrolled in this study.

\section{Surgical procedure and pathological findings}

The R-category and extent of dissection were determined by the Japanese Classification of Gastric Carcinoma, 3rd English edition, and the JGCA guidelines [13]. In principle, D2 gastrectomy was adopted for T2-T4 disease, whereas D1+ was selected for $\mathrm{T} 1$ cancer. Resected specimens were examined histopathologically, and were staged according to the International Union Against Cancer (UICC) TNM, 7th edition [14].

\section{Perioperative care}

All patients were treated using the same enhanced recovery after surgery (ERAS) protocol for gastric cancer surgery. The details have been reported previously [15]. In brief, the patients were allowed to eat until midnight of the day before surgery and were allowed to drink an oral rehydration solution until $3 \mathrm{~h}$ before surgery. The nasogastric tube was removed immediately after surgery. Patients were encouraged to sit out of bed for more than $6 \mathrm{~h}$ on postoperative day (POD) 1. Oral intake was started on POD 2, and patients started to eat solid food on POD3. The patients were also encouraged to walk the length of the ward. The patients were discharged when they had achieved adequate pain relief and soft food intake, had returned to their preoperative mobility level, and had normal laboratory data on POD 7. The patients were removed from ERAS perioperative care when they developed complications for which they required special care.

\section{Definition of postoperative complications}

Complications that occurred during hospitalization or within 30 days after surgery were retrospectively extracted from the patient record and classified as grade 2-5 according to the Clavien-Dindo classification [16, 17]. Grade 1 complications were not evaluated to exclude the possibility of a description bias in the patient records.

\section{Measurements of preoperative muscle strength and lean body mass}

In our division, the nutrition support team has initiated prospective measurement of HGS and BIA since 2011. All data were recorded prospectively. Muscle strength was evaluated by measuring hand grip strength (HGS) using a hand-held dynamometer (Scientific Instruments, Niigata, Japan). The hand grip strength was expressed in kilograms $(\mathrm{kg})$ at baseline. With the patient in a standing position with both hands lowered, the grip size was adjusted so that the subject felt comfortable while squeezing the grip. The subjects were then instructed and verbally encouraged to squeeze the hand grip as hard as they could. Two trials were performed in both hands, and the mean of the two hands' grip scores was recorded as patient's score. Lean body mass (LBM) was estimated from a bioelectrical impedance analysis using a MC-180 Body Composition Analyzer (Tanita, Tokyo, Japan). The LBM was converted to an LBM index $\left(\mathrm{kg} / \mathrm{m}^{2}\right)$ by dividing the LBM by the height in meters squared.

Each cutoff value was determined as the gender-specific lowest $20 \%$ of the distribution of each measurement based on previously published studies $[8,18]$.

\section{Statistical analyses}

The data were statistically analyzed using the Wilcoxon test and the $\chi^{2}$ test. The impact of the LBM and HGS on 
postoperative morbidity were examined using univariate and multivariate logistic regression analyses. Values of $P<0.05$ were considered to indicate statistical significance. All statistical analyses were performed using the Dr. SPSS II software program, version 11.0.1 J for Windows (SPSS, Chicago, IL, USA).

\section{Results}

A total of 315 patients underwent D2 or D1+ gastrectomy for gastric carcinoma during this period. Among them, 310 underwent R0 or R1 resection. Seventeen patients were excluded because of double cancer or a lack of preoperative measurements of muscle strength and LBM. Therefore, 293 patients were finally entered into the study. The clinical and pathological characteristics are outlined in Table 1. Twenty-two patients $(7.5 \%)$ were more than 80 years old. The majority $(212,72.4 \%)$ of the patients had an ASA score of 2 in this series. Five patients (1.7\%) had a body mass index greater than $30 \mathrm{~kg} / \mathrm{m}^{2}$.

\section{Surgical procedure and pathological findings}

The surgical approach was open surgery in 205 patients and laparoscopic surgery in 88. A total of 132 patients (45.1\%) underwent D2 lymphadenectomy, 122 patients $(41.6 \%)$ underwent total gastrectomy, and 53 (18.1\%) underwent splenectomy. Overall, 172 patients (58.7\%) had early-stage disease.

The cutoff values of the LBM index were determined to be $17.0 \mathrm{~kg} / \mathrm{m}^{2}$ in men and $14.8 \mathrm{~kg} / \mathrm{m}^{2}$ in women, whereas those of HGS were as $27.5 \mathrm{~kg}$ in men and $16.2 \mathrm{~kg}$ in women (Fig. 1).

\section{Risk factors for postoperative complications}

Of the 293 patients who underwent surgery for gastric cancer, 39 patients developed a grade 2 or more severe complication, resulting in a postoperative morbidity rate of $13.3 \%$. No surgical mortality was observed.

Among the background factors shown in Table 1, body mass index, ASA classification, surgical approach, extent of gastrectomy, splenectomy and/or pancreatectomy, extent of nodal dissection, LBM index, and HGS were selected as possible risk factors for surgical morbidity. Each factor was categorized as shown in Table 3 after considering the theoretical background of each factor. The univariate analysis showed that male gender, total gastrectomy, splenectomy, and a low HGS were significant risk factors for morbidity. A low LBM index was not a significant risk factor (Table 2). A low HGS (HR 2.457, 1.098-5.494, $P=0.029$ ), male gender (HR 2.610,
Table 1 Patient characteristics $(n=293)$

\begin{tabular}{|c|c|}
\hline Age, median (range) & $66(33-85)$ years \\
\hline Gender, M/F & $192 / 101$ \\
\hline Body mass index, median (range) & $22.0(13.9-34.8) \mathrm{kg} / \mathrm{m}^{2}$ \\
\hline \multicolumn{2}{|l|}{ ASA classification } \\
\hline 1 & 75 \\
\hline 2 & 212 \\
\hline 3 & 6 \\
\hline LBM index, median (range) & $16.4(12.7-21.6) \mathrm{kg} / \mathrm{m}^{2}$ \\
\hline Hand grip strength, median (range) & $28.6(11.2-75.5) \mathrm{kg}$ \\
\hline \multicolumn{2}{|l|}{ Site of tumor } \\
\hline Upper third & 68 \\
\hline Middle third & 120 \\
\hline Lower third & 107 \\
\hline Tumor size, mean (range) & $30(4-190) \mathrm{mm}$ \\
\hline \multicolumn{2}{|l|}{ Histological type } \\
\hline Differentiated & 120 \\
\hline Undifferentiated & 173 \\
\hline \multicolumn{2}{|l|}{ Surgical approach } \\
\hline Open surgery & 205 \\
\hline Laparoscopic surgery & 88 \\
\hline \multicolumn{2}{|l|}{ Surgical procedure } \\
\hline Total gastrectomy & 122 \\
\hline Subtotal gastrectomy & 171 \\
\hline Length of operation time, median (range) & $245(95-579) \min$ \\
\hline \multicolumn{2}{|l|}{ Combined resection } \\
\hline Spleen & 53 \\
\hline Pancreas & 2 \\
\hline \multicolumn{2}{|l|}{ Extended lymphadenectomy } \\
\hline $\mathrm{D} 1+$ & 161 \\
\hline D2 & 132 \\
\hline \multicolumn{2}{|l|}{ Pathological stage } \\
\hline I & 149 \\
\hline II & 39 \\
\hline III & 91 \\
\hline IV & 14 \\
\hline
\end{tabular}

ASA American Society of Anesthesiologists, $L B M$ lean body mass

1.052-6.493, $P=0.038$ ) and total gastrectomy (HR 2.747, $1.112-6.711, P=0.027)$ remained significant in the multivariate analysis (Table 2).

We then examined the incidence of complications by stratifying the patients into two categories based on the HGS: a low HGS group $(n=54)$ and a high HGS group $(n=239)$. The morbidity rates were $22.2 \%$ in the low HGS group and $11.3 \%$ in the high HGS group, which was a statistically significant difference $(P=0.004$; Table 3$)$. The univariate analysis showed that pneumonia was significantly more common in the low HGS group than the high HGS group (Table 3). The other complications did not differ significantly between the two groups. 


\section{(a) LBM index $\left(\mathrm{kg} / \mathrm{m}^{2}\right)$ for males}

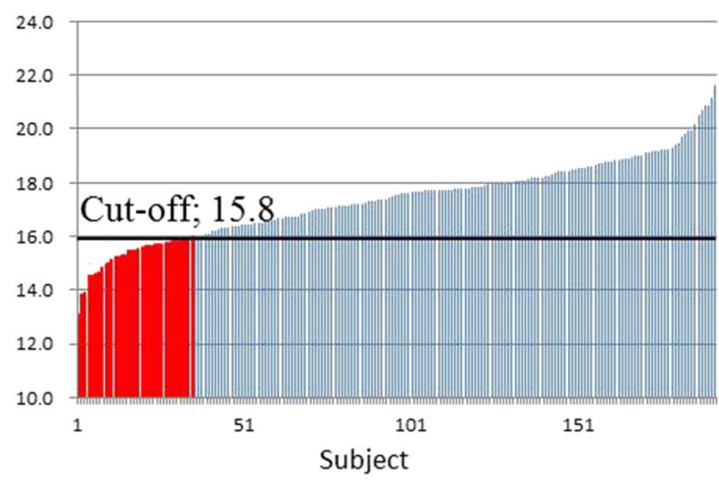

(c) LBM index $\left(\mathrm{kg} / \mathrm{m}^{2}\right)$ for females

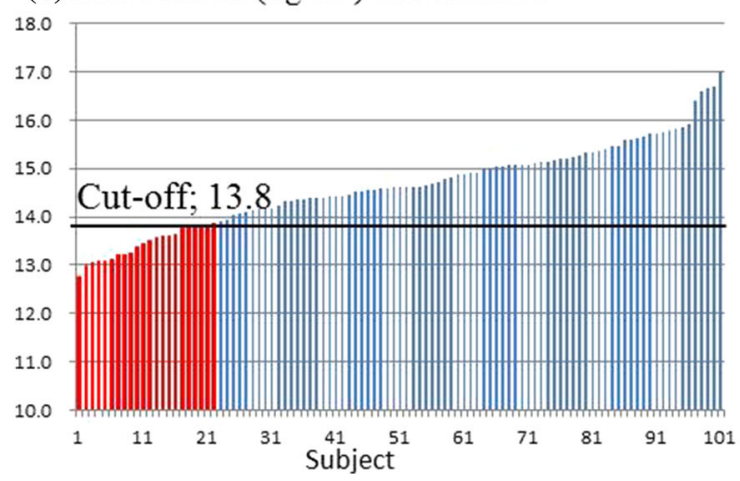

Fig. 1 Distribution of gender-specific lean body mass (LBM) index $\left(\mathrm{kg} / \mathrm{m}^{2}\right)$ and hand grip strength $(H G S, \mathrm{~kg})$. Cutoff points were defined as the lowest gender-specific 20th percentile of each value. Subjects

\section{Discussion}

This report focused on the impact of sarcopenia, characterized by muscle strength or mass, on postoperative complications after gastrectomy for gastric cancer. This report revealed, for the first time, that low muscle strength, but not decreased LBM index, was an independent risk factor for morbidity after gastric cancer surgery. The value of hand grip strength as a risk factor for morbidity should be examined in future prospective studies.

In this study, we measured the HGS as an index of muscle strength. Lauretani et al. previously examined upper and lower extremity muscle strength and cross-sectional calf muscle area in the healthy elderly. They found that HGS was strongly related to knee extension torque and calf cross-sectional muscle area [19]. Norman et al. also reported that the HGS correlated well with the results of other muscle function tests, such as knee extension strength, in cancer patients [20]. The European Working Group on Sarcopenia in Older People (EWGSOP) recommended that the HGS should be used as a measure of muscle strength when diagnosing sarcopenia [16]. Based on these previous reports, we evaluated the HGS as a measure of muscle strength in the present study. (b) HGS (kg) for males

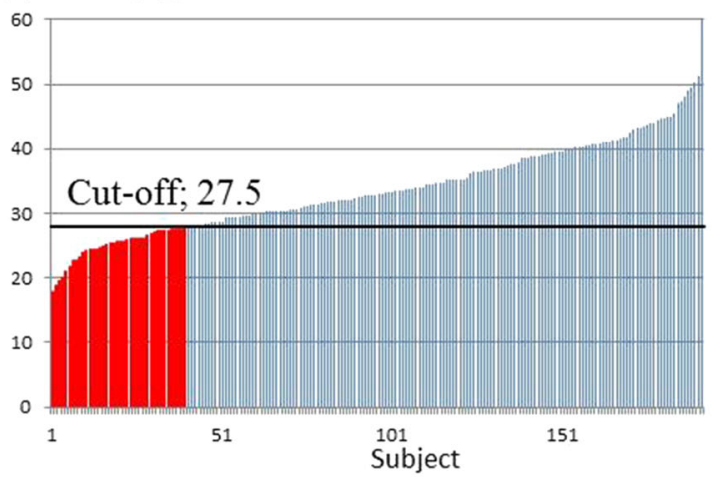

(d) HGS (kg) for females

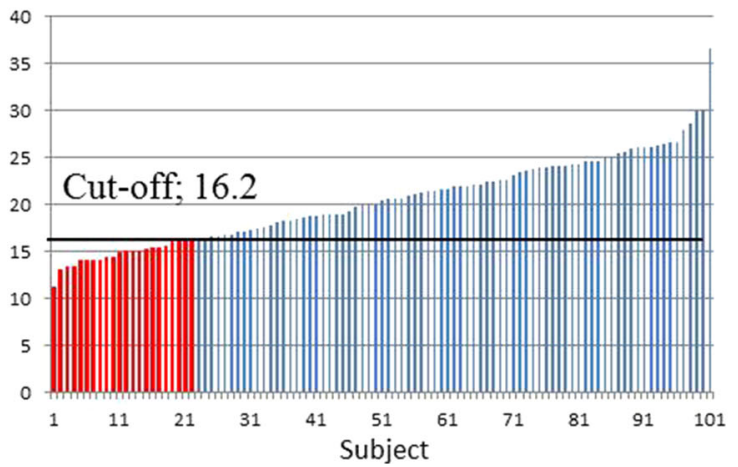

with lower values are shown with red bars; those with higher values are indicated with blue bars

As a result, it was clearly demonstrated that a low HGS was a significant independent risk factor for postoperative complications. As already mentioned, HGS could be a surrogate measure for knee extension strength. Patients with a low HGS were considered to have low knee extension strength, which suggests that these patients had low walking ability. In this series, all patients received the same ERAS protocol, in which patients were encouraged to sit out of bed for more than $6 \mathrm{~h}$ on postoperative day 1 and to walk the length of the ward on day 2 after surgery. Such early ambulation is likely to be difficult for the patients with low walking ability. The amount or frequency of ambulation may be inhibited in these patients.

In this study, only the incidence of pneumonia was significantly different between the low and high HSG groups among all surgical complications examined. Moreover, HGS was only a significant independent risk factor for pneumonia after surgery. On the other hand, the rates of ileus, delirium, and venous thromboembolism were not significantly different. Thus, low walking ability does not seem to be sufficient to explain why a low HGS was associated with an increased risk of surgical complications. However, Norman et al. previously reported that HGS was correlated with peak expiratory flow [20]. Thus, patients 
Table 2 Results of univariate and multivariate analyses of risk factors for postoperative complications (grade $\geq 2$ )

\begin{tabular}{|c|c|c|c|c|c|c|}
\hline \multirow[t]{2}{*}{ Variable/category } & \multirow{2}{*}{ Absent $(n=254)$} & \multirow[t]{2}{*}{ Present $(n=39)$} & \multicolumn{2}{|c|}{ Univariate } & \multicolumn{2}{|c|}{ Multivariate } \\
\hline & & & OR & $P$ value & OR & $P$ value \\
\hline \multicolumn{7}{|l|}{ Age (years) } \\
\hline$<66$ & 126 & 17 & 1 & & & \\
\hline$\geq 66$ & 128 & 22 & 1.033 & 0.497 & & \\
\hline \multicolumn{7}{|l|}{ Gender } \\
\hline Female & 94 & 7 & 1 & & 1 & \\
\hline Male & 160 & 32 & 2.709 & 0.019 & 2.610 & 0.038 \\
\hline \multicolumn{7}{|c|}{ Body mass index $\left(\mathrm{kg} / \mathrm{m}^{2}\right)$} \\
\hline$<25$ & 209 & 31 & 1 & & & \\
\hline$\geq 25$ & 45 & 8 & 1.204 & 0.657 & & \\
\hline \multicolumn{7}{|l|}{ ASA classification } \\
\hline 1 & 69 & 6 & 1 & & & \\
\hline $2-3$ & 185 & 33 & 2.040 & 0.166 & & \\
\hline \multicolumn{7}{|l|}{ Surgical approach } \\
\hline Laparoscopic surgery & 77 & 9 & 1 & & & \\
\hline Open surgery & 175 & 30 & 1.477 & 0.219 & & \\
\hline \multicolumn{7}{|l|}{ Surgical procedure } \\
\hline Subtotal gastrectomy & 160 & 11 & 1 & 1 & & \\
\hline Total gastrectomy & 94 & 28 & 4.287 & 0.001 & 2.747 & 0.027 \\
\hline \multicolumn{7}{|c|}{ Splenectomy and/or pancreatectomy } \\
\hline Absent & 217 & 23 & 1 & 1 & & \\
\hline Present & 37 & 16 & 4.099 & 0.001 & 2.222 & 0.075 \\
\hline \multicolumn{7}{|l|}{ Dissected lymph nodes } \\
\hline $\mathrm{D} 1+$ & 144 & 17 & 1 & & & \\
\hline D2 & 110 & 22 & 1.706 & 0.166 & & \\
\hline \multicolumn{7}{|l|}{ LBM index $\left(\mathrm{kg} / \mathrm{m}^{2}\right)$} \\
\hline$\geq$ GSL $20 \%$ & 204 & 31 & 1 & & & \\
\hline$<$ GSL $20 \%$ & 50 & 8 & 1.048 & 1.000 & & \\
\hline \multicolumn{7}{|l|}{ Hand grip strength (kg) } \\
\hline$\geq$ GSL $20 \%$ & 212 & 27 & 1 & 1 & & \\
\hline$<$ GSL $20 \%$ & 42 & 12 & 2.254 & 0.044 & 2.457 & 0.029 \\
\hline
\end{tabular}

GSL gender-specific lowest 20th percentile, ASA American Society of Anesthesiologists, LBM lean body mass, $O R$ odds ratio with a low HGS are considered to have a low peak expiratory flow, although expiratory flow was not examined in our study. Further supporting the significance of the peak expiratory flow, Windsor et al. reported that a low value was one of the risk factors for pulmonary complications [21]. The peak expiratory flow is an individual's maximum speed of expiration, which is used to monitor the individual's ability to breathe out air, and is proportional to respiratory muscle strength [22]. It is also well known that respiratory muscle weakness decreases the vital capacity of the lungs [23]. Although the exact mechanism(s) are unclear, a low HGS may be related to pulmonary complications from the low peak expiratory flow or low respiratory muscle strength. On the other hand, sarcopenia is closely related to host immunity. The decreased expression of myokines observed in patients with sarcopenia could decrease NK cell activity [24, 25], which would increase the risk of infectious complications. Further studies are necessary to clarify how sarcopenia increases the risk of infectious complications after gastric cancer surgery.

In the present study, the LBM as measured by BIA was not a significant risk factor for surgical morbidity, which was contradictory to the previous studies evaluating the slices examined by computed tomography (CT) $[11,12]$. This discrepancy could be explained by the difference in the characteristics of the cohort and the methodological difference. Because T1 is a major population of our cohort and those undergoing R2 resection necessitated by far-advanced disease were excluded from the study, it is unlikely that our series has many patients who have muscle mass 
Table 3 Postoperative complications

\begin{tabular}{lcllccc}
\hline & Total & Low HGS $(n=54)$ & High HGS $(n=239)$ & OR & $95 \%$ CI & $P$ value \\
\hline Anastomotic leakage & $12(4.3 \%)$ & $3(5.6 \%)$ & $9(3.8 \%)$ & 1.503 & $0.393-5.749$ & 0.468 \\
Anastomotic stenosis & $1(0.3 \%)$ & 0 & $1(0.4 \%)$ & 0.988 & $0.988-1.004$ & 1.000 \\
Bleeding & $2(0.7 \%)$ & 0 & $2(0.8 \%)$ & 0.992 & $0.980-1.003$ & 1.000 \\
Intraabdominal abscess & $4(1.2 \%)$ & $2(3.7 \%)$ & $2(0.8 \%)$ & 4.558 & $0.628-33.103$ & 0.156 \\
Pancreatic fistula & $7(2.4 \%)$ & $2(3.7 \%)$ & $5(2.1 \%)$ & 1.800 & $0.340-9.534$ & 0.617 \\
Pancreatitis & $2(0.7 \%)$ & 0 & $2(0.8 \%)$ & 0.992 & $0.980-1.003$ & 1.000 \\
Delayed gastric emptying & $1(0.3 \%)$ & 0 & $1(0.4 \%)$ & 0.996 & $0.988-1.004$ & 1.000 \\
Ileus & $3(1.0 \%)$ & 0 & $3(1.2 \%)$ & 0.988 & $0.974-1.002$ & 1.000 \\
Lymphorrhea & $1(0.3 \%)$ & 0 & $1(0.4 \%)$ & 0.996 & $0.988-1.004$ & 1.000 \\
Pneumonia & $5(1.7 \%)$ & $4(7.1 \%)$ & $1(0.4 \%)$ & 19.040 & $2.084-173.98$ & 0.005 \\
Atelectasis & $1(0.3 \%)$ & $1(2.0 \%)$ & 0 & 1.020 & $0.981-1.060$ & 0.174 \\
Any complication & $39(13.3 \%)$ & $12(23.5 \%)$ & $27(11.2 \%)$ & 2.254 & $1.058-4.802$ & 0.044 \\
\hline
\end{tabular}

$H G S$ hand grip strength, $O R$ odds ratio, $C I$ confidence interval

depletion caused by cancer progression, which may be the reason why HGS, but not LBM, was the risk factor for morbidity. Another possibility is the differences in the methods used to evaluate the muscle, with the LBM being indirectly measured using BIA and directly measured using body imaging techniques, such as CT. In the BIA method, the LBM is measured by bioelectrical impedance, which is affected by the amount of water. The LBM represents the weight of muscles, bones, ligaments, tendons and internal organs. In particular, the results obtained by BIA have been demonstrated to have poor concordance with the direct measurement of muscle LBM by dual energy X-ray-based measurements in malnourished patients with advanced or metastatic cancer [26, 27]. However, more than half of the present cohort had stage I cancer, and the nutritional status of these patients would not have been affected by the cancer itself. On the other hand, CT and MRI can directly evaluate muscle LBM. Shen et al. measured the total area of the abdominal skeletal muscle, and demonstrated that the total abdominal muscle area could be an accurate surrogate for the whole body muscle [28]. Direct measurement of muscle mass is reliable but time consuming, and we cannot ignore X-ray exposure in the case of CT, whereas BIA is safe and convenient. Moreover, our recent preliminary study showed a strong relationship between LBM calculated by a MC-180 Body Composition Analyzer (Tanita, Tokyo, Japan) and area of the psoas muscle mass evaluated by $\mathrm{CT}$ in preoperative gastric cancer patients (data not shown). Based on this, we selected the BIA method for the present study.

We defined cutoff points at the gender-specific lower 20th percentiles of the values in this cohort. The EWGSOP recommends the use of normative, rather than other predictive reference populations, with cutoff points at two standard deviations below the mean reference value. On the other hand, it has been suggested that there are no true cut points for sarcopenia, and there are various options that can be used to define subnormal values for sarcopenia [29]. Baumgartner et al. defined two standard deviations below the mean LBM index of young male and female reference groups as the gender-specific cut point for sarcopenia [30]. Delmonico et al. and Newman et al. used the genderspecific 20th percentile of their study group and young adults in the study group as the cutoff point for sarcopenia $[8,18]$. Ideally, specific cutoff points for sarcopenia should be determined for each specific patient population and race. There have been a few reports that defined a cutoff point for sarcopenia in Japanese [31, 32]. Based on these studies, we defined the cutoff point as the gender-specific 20th percentile in this study.

HGS is a convenient method to evaluate the muscle strength; however, it is associated with some limitations. The HGS is only an indicator of upper limb strength and cannot replace an accurate assessment of the activities of daily living, lower extremity strength, or walking speed in fragile populations such as the elderly or patients with neuromuscular disease. Previously, Prado et al. reported that patients with sarcopenia had poorer functional status (PS score 2-4) compared with patients who did not have sarcopenia [33]. Furthermore, Chukwuemeka et al. reported that a higher ECOG PS score is an independent predictor for postoperative morbidity [34]. Considering these reports, the impact of low HGS on morbidity might be weakened and ECOG PS might be selected as a risk factor if patients with PS above 2 are included. HGS would have a great advantage to select patients with good performance status but high risk for morbidity. Previous studies for other upper abdominal surgery such as hepatocellular carcinoma, pancreas cancer, and metastatic liver tumor demonstrated that sarcopenia was a risk factor for postoperative 
complication [12, 35, 36]. However, no report showed a correlation of sarcopenia and respiratory complications, which is different from the present study. Gastric cancer surgery has a risk of dysphagia because of esophageal reflux induced by nodal dissection or reconstruction, which is different from other upper abdominal surgery. The risk of pneumonia is highest in gastric cancer surgery patients among all upper abdominal surgical populations. Thus, specific infectious complications other than pneumonia could be related with sarcopenia to other upper abdominal surgery.

In conclusion, a low HGS was found to be a significant risk factor for morbidity after gastric cancer surgery. A low HGS significantly increased postoperative pneumonia. Because this is a retrospective study, selection bias could not be ruled out. Moreover, not all the treatment is specifically determined by the protocol. Thus, the value of HGS as a surgical risk factor should be examined in future prospective studies.

Acknowledgments The authors express their sincere gratitude to Ms. Rika Takahashi for her excellent data management and to the members of the nutritional support team for measurement of hand grip strength and body impedance analysis.

\section{Compliance with ethical standards}

Conflict of interest The authors declare that they have no conflicts of interest related to the contents of this manuscript.

Human rights statement and informed consent All procedures followed were in accordance with the ethical standards of the responsible committee on human experimentation (institutional and national) and with the Helsinki Declaration of 1964 and later versions. Informed consent or a substitute for it was obtained from all patients for being included in the study.

\section{References}

1. Sano T, Sasako M, Yamamoto S, Nashimoto A, Kurita A, Hiratsuka M, et al. Gastric cancer surgery: morbidity and mortality results from a prospective randomized controlled trial comparing D2 and extended para-aortic lymphadenectomy-Japan Clinical Oncology Group study 9501. J Clin Oncol. 2004;22:2767-73.

2. Songun I, Putter H, Kranenbarg EM, Sasako M, van de Velde CJ. Surgical treatment of gastric cancer: 15-year follow-up results of the randomised nationwide Dutch D1D2 trial. Lancet Oncol. 2010;11:439-49.

3. Yu J, Hu J, Huang C, Ying M, Peng X, Wei H, et al. The impact of age and comorbidity on postoperative complications in patients with advanced gastric cancer after laparoscopic D2 gastrectomy: results from the Chinese Laparoscopic Gastrointestinal Surgery Study (CLASS) group. Eur J Surg Oncol. 2013;39:1144-9.

4. Sugisawa N, Tokunaga M, Tanizawa Y, Bando E, Kawamura T, Terashima M. Intra-abdominal infectious complications following gastrectomy in patients with excessive visceral fat. Gastric Cancer. 2012;15:206-12.
5. Jeong SH, Ahn HS, Yoo MW, Cho JJ, Lee HJ, Kim HH, et al. Increased morbidity rates in patients with heart disease or chronic liver disease following radical gastric surgery. J Surg Oncol. 2010;101:200-4.

6. Tsujinaka T, Sasako M, Yamamoto S, Sano T, Kurokawa Y, Nashimoto A, et al. Influence of overweight on surgical complications for gastric cancer: results from a randomized control trial comparing D2 and extended para-aortic D3 lymphadenectomy (JCOG9501). Ann Surg Oncol. 2007;14:355-61.

7. Antoun S, Lanoy E, Iacovelli R, Albiges-Sauvin L, Loriot Y, Merad-Taoufik M, et al. Skeletal muscle density predicts prognosis in patients with metastatic renal cell carcinoma treated with targeted therapies. Cancer. 2013;119(18):3377-84.

8. Delmonico MJ, Harris TB, Lee JS, Visser M, Nevitt M, Kritchevsky SB, et al. Alternative definitions of sarcopenia, lower extremity performance, and functional impairment with aging in older men and women. J Am Geriatr Soc. 2007;55:769-74.

9. Englesbe MJ, Patel SP, He K, Lynch RJ, Schaubel DE, Harbaugh C, et al. Sarcopenia and mortality after liver transplantation. J Am Coll Surg. 2010;211:271-8.

10. Kaido T, Ogawa K, Fujimoto Y, Ogura Y, Hata K, Ito T, et al. Impact of sarcopenia on survival in patients undergoing living donor liver transplantation. Am J Transplant. 2013;13:1549-56.

11. Lieffers JR, Bathe OF, Fassbender K, Winget M, Baracos VE. Sarcopenia is associated with postoperative infection and delayed recovery from colorectal cancer resection surgery. Br J Cancer. 2012;107:931-6.

12. Peng PD, van Vledder MG, Tsai S, de Jong MC, Makary M, Ng J, et al. Sarcopenia negatively impacts short-term outcomes in patients undergoing hepatic resection for colorectal liver metastasis. HPB (Oxf). 2011;13:439-46.

13. Japanese gastric cancer treatment guidelines. ver. 3. Gastric Cancer. 2010;2011(14):113-23.

14. Sobin LH, Gospodarowicz MK, Wittekind C. TNM classification of malignant tumours. New York: Wiley; 2011.

15. Yamada $T$, Hayashi $T$, Cho $H$, Yoshikawa $T$, Taniguchi $H$, Fukushima R, et al. Usefulness of enhanced recovery after surgery protocol as compared with conventional perioperative care in gastric surgery. Gastric Cancer. 2011;15(1):34-41.

16. Clavien PA, Barkun J, de Oliveira ML, Vauthey JN, Dindo D, Schulick RD, et al. The Clavien-Dindo classification of surgical complications: five-year experience. Ann Surg. 2009;250:187-96.

17. Dindo D, Demartines N, Clavien PA. Classification of surgical complications: a new proposal with evaluation in a cohort of 6336 patients and results of a survey. Ann Surg. 2004;240:205-13.

18. Newman AB, Kupelian V, Visser M, Simonsick E, Goodpaster B, Nevitt M, et al. Sarcopenia: alternative definitions and associations with lower extremity function. $\mathrm{J}$ Am Geriatr Soc. 2003;51:1602-9.

19. Lauretani F, Russo CR, Bandinelli S, Bartali B, Cavazzini C, Di Iorio $\mathrm{A}$, et al. Age-associated changes in skeletal muscles and their effect on mobility: an operational diagnosis of sarcopenia. J Appl Physiol. 2003;95:1851-60.

20. Norman K, Stobaus N, Smoliner C, Zocher D, Scheufele R, Valentini L, et al. Determinants of hand grip strength, knee extension strength and functional status in cancer patients. Clin Nutr. 2010;29:586-91.

21. Windsor JA, Hill GL. Risk factors for postoperative pneumonia. The importance of protein depletion. Ann Surg. 1988;208:209-14.

22. Arora NS, Rochester DF. Respiratory muscle strength and maximal voluntary ventilation in undernourished patients. Am Rev Respir Dis. 1982;126:5-8. 
23. Bosnak-Guclu M, Gunduz AG, Nazliel B, Irkec C. Comparison of functional exercise capacity, pulmonary function and respiratory muscle strength in patients with multiple sclerosis with different disability levels and healthy controls. J Rehabil Med. 2012;44:80-6.

24. Lutz CT. Quinn LS Sarcopenia, obesity, and natural killer cell immune senescence in aging: altered cytokine levels as a common mechanism. Aging (Albany NY). 2012;4:535-46.

25. Woods JL, Iuliano-Burns S, Walker KZ. Immunological and nutritional factors in elderly people in low-level care and their association with mortality. Immun Ageing. 2013;10:32.

26. Trutschnigg B, Kilgour RD, Reinglas J, Rosenthall L, Hornby L, Morais JA, et al. Precision and reliability of strength (Jamar vs. Biodex handgrip) and body composition (dual-energy X-ray absorptiometry vs. bioimpedance analysis) measurements in advanced cancer patients. Appl Physiol Nutr Metab. 2008;33:1232-9.

27. Mourtzakis M, Prado CM, Lieffers JR, Reiman T, McCargar LJ, Baracos VE. A practical and precise approach to quantification of body composition in cancer patients using computed tomography images acquired during routine care. Appl Physiol Nutr Metab. 2008;33:997-1006.

28. Shen W, Punyanitya M, Wang Z, Gallagher D, St-Onge MP, Albu $\mathrm{J}$, et al. Total body skeletal muscle and adipose tissue volumes: estimation from a single abdominal cross-sectional image. J Appl Physiol. 2004;97:2333-8.

29. Cruz-Jentoft AJ, Baeyens JP, Bauer JM, Boirie Y, Cederholm T, Landi F, et al. Sarcopenia: European consensus on definition and diagnosis. Report of the European Working Group on Sarcopenia in Older People. Age Ageing. 2010;39:412-23.

30. Baumgartner RN, Koehler KM, Gallagher D, Romero L, Heymsfield SB, Ross RR, et al. Epidemiology of sarcopenia among the elderly in New Mexico. Am $\mathrm{J}$ Epidemiol. 1998;147:755-63.

31. Tanimoto Y, Watanabe M, Sun W, Tanimoto K, Shishikura K, Sugiura Y, et al. Association of sarcopenia with functional decline in community-dwelling elderly subjects in Japan. Geriatr Gerontol Int. 2013;13:958-63.

32. Tanimoto Y, Watanabe M, Sun W, Hirota C, Sugiura Y, Kono R, et al. Association between muscle mass and disability in performing instrumental activities of daily living (IADL) in community-dwelling elderly in Japan. Arch Gerontol Geriatr. 2012;54:e230-3.

33. Prado CM, Lieffers JR, McCargar LJ, Reiman T, Sawyer MB, Martin L, et al. Prevalence and clinical implications of sarcopenic obesity in patients with solid tumours of the respiratory and gastrointestinal tracts: a population-based study. Lancet Oncol. 2008;9:629-35.

34. Ihemelandu CU, McQuellon R, Shen P, Stewart JH, Votanopoulos K, Levine EA. Predicting postoperative morbidity following cytoreductive surgery with hyperthermic intraperitoneal chemotherapy (CS+HIPEC) with preoperative FACT-C (Functional Assessment of Cancer Therapy) and patient-rated performance status. Ann Surg Oncol. 2013;20:3519-26.

35. Valero V 3rd, Amini N, Spolverato G, Weiss MJ, Hirose K, Dagher NN, et al. Sarcopenia adversely impacts postoperative complications following resection or transplantation in patients with primary liver tumors. J Gastrointest Surg. 2015;19:272-81.

36. Amini N, Spolverato G, Gupta R, Margonis GA, Kim Y, Wagner $\mathrm{D}$, et al. Impact total psoas volume on short- and long-term outcomes in patients undergoing curative resection for pancreatic adenocarcinoma: a new tool to assess sarcopenia. J Gastrointest Surg. 2015;19:1593-602. 